\title{
GERANIO PRATENSI-CIRSIETUM CANI ASS. NOVA PÎNZARU, IONIŢA \& JARDAN (FILIPENDULION SEGAL EX WESTHAFF ET DEN HELD 1969) IN THE REPUBLIC OF MOLDOVA
}

\author{
$\underline{\text { Pavel PINZZARU }}^{1 *}$, Olga IONIŢA ${ }^{1}$, Natalia JARDAN $^{2}$ \\ 1 “Alexandru Ciubotaru” National Botanical Garden (Institute), Chișinău - Republic of \\ Moldova \\ 2 “Codii” Reserve, Lozova commune, Strășeni district - Republic of Moldova \\ ${ }^{*}$ Corresponding author. E-mail: p_panzaru@yahoo.it
}

\begin{abstract}
The phytocoenoses of Cirsium canum (L.) All. with Geranium pratense L., occurring on the Central Moldavian Plateau, are described in this article. Based on 26 relevés, the authors propose another association for science - Geranio pratensi-Cirsietum cani ass. nova Pînzaru, Ionița et Jardan of the alliance Filipendulion ulmariae Segal ex Westhoff et Den Held 1969, order Molinietalia caeruleae Koch 1926, class MOLINIO-ARRHENATHERETEA Tx. 1937.
\end{abstract}

Keywords: Geranio pratensi-Cirsietum cani ass. nova, characteristics plant ofspecies, ecology, range, Republic of Moldova.

\section{Introduction}

Spear thistle or Queen Anne's thistle (Cirsium canum (L.) All., Figure 1) - a perennial species, geophyte, East-European, meso-hygrophilic, is characteristic of wet meadows from lowland to mountainous areas, included in the order Molinietalia caeruleae W. Koch 1926 [AESCHIMANN \& al. 2004; SÂRBU \& al. 2013]. The Central European

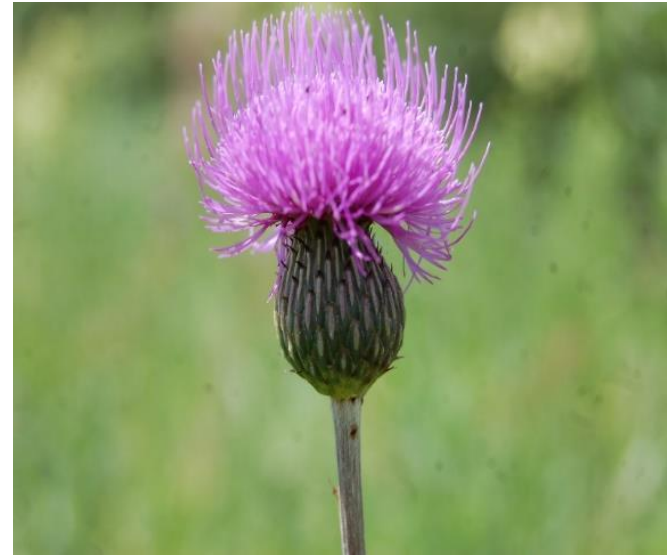

Figure 1. Cirsium canum (L.) All. plant communities with Cirsium canum are included in the associations: Cirsio caniFestucetum pratensis Májovsky et Ruzicková 1973 of the alliance Deschampsion cespitosae Horvatić 1930 (= Alopecurion pratensis Passarge 1964), Scirpo sylvatici-Cirsietum cani BálátováTulačová 1973 of the alliance Calthion palustris R.Tx. 1937, Angelico sylvestrisCirsietum cani P. Burescu 1998 corr. Chifu et Zamfirescu 2014 of the alliance Calthion palustris R. Tx. 1937 [BĂDĂRĂU \& ALEC-FARCAS, 2010; CHIFU \& al. 2014; COLDEA \& al. 2012; HÁJKOVÁ, 2010].

The vegetation of floodplain grasslands in the Republic of Moldova has been described in more detail in the monograph of the botanist Ştefan Lazu, where he has also mentioned an association with Cirsium canum (L.) All. - Cirsietum cani Tx. 1951, with a short characterization based on 4 relevés, grouped 
in the alliance Agrostion stoloniferae Soó 1933 [LAZU, 2014]. At that time, in 2014, the association Cirsietum cani R.Tx. et Preising 1951 was considered as a synonym of the association Angelico sylvestris-Cirsietum cani P. Burescu 1998 corr. Chifu et Zamfirescu 2014 of the alliance Calthion palustris R.Tx. 1937 [CHIFU \& al. 2014]. The new classification of the vegetation in Europe [MUCINA \& al. 2016] does not indicate the alliance Agrostion stoloniferae Soó (1933) 1971 [BĂDĂRĂU \& ALEC-FARCAS, 2010], but only Agrostion stoloniferae Görs 1966, which is synonymous with the alliance Potentillion anserinae Tx. 1947.

The phytocoenoses of Cirsium canum (L.) All. with Geranium pratense L. recorded in the floodplains of rivers on the Central Moldavian Plateau are described in this article.

\section{Materials and methods}

The phytocoenological research was carried out in 2018-2019. The research methodology adopted is that of the better write "The Zürich-Montpellier School" founded by Braun-Blanquet [BRAUN-BLANQUET, 1964]. The area of a relevé was $100 \mathrm{~m}$ [CRISTEA, 2004]. The plant species nomenclature is presented in accordance with recent publications [PÎNZARU \& SÎRBU, 2016]. Air temperature and atmospheric precipitation - according to the Atlas of Climate Resources of the Republic of Moldova [NEDEALCOV \& al. 2013].

\section{Results and discussions}

The plant communities of Cirsium canum, Geranium pratense, Inula helenium and other accompanying species, which occur on slightly alkaline stratified alluvial soils, in the floodplains of rivers on the Central Moldavian Plateau, are tall (the upper layer is about 140$180 \mathrm{~cm}$ in height) and contain a group of species that is characteristic of the alliance Filipendulion ulmariae Segal ex Westhoff et Den Held 1969, which is the reason why we include them in this alliance.

The alliance Filipendulion ulmariae Segal ex Westhoff et Den Held 1969 consists of herbaceous, meso-hygrophilic, tall plants, which occur in river valleys and valleys between hills or between mountains, on alluvial soils, which are moist and rich in nutrients. The characteristic plant species of the alliance are: Filipendula ulmaria, Geranium palustre, Valeriana officinalis, Calystegia sepium, Lysimachia vulgaris, Lythrum salicaria, Mentha longifolia, Euphorbia palustris, Epilobium hirsutum, E. parviflorum, Petasites hybridus Stachys palustris, Symphytum officinale, Poa palustris, Hypericum tetrapterum [CHIFU \& al. 2014; COLDEA \& al. 2012; HÁJKOVÁ, 2010; PÎNZARU, 1996; SÂRBU \& al. 2013].

The described associations, occurring in Moldova, Petasitetum hybridi (Dostal 1933) Soó 1940 [LAZU, 2014; PÎNZARU, 1996] and Filipendulo-Geranietum palustris W. Koch 1926 [LAZU, 2014], previously included in the alliance Filipendulo-Petasition Br.-B1. 1947, are now grouped in the alliance Filipendulion ulmariae Segal ex Westhoff et Den Held 1969. The alliance Filipendulo-Petasition Br.-Bl. ex Duvigneaud 1949 contains a group of associations of the submontane-montane layer in Western and Central Europe [MUCINA, 2016]. In Romania it hasn't been detected [CHIFU \& al. 2014; COLDEA \& al. 2012].

The description of the association of Cirsietum cani Tx. 1951 made by LAZU (2014, tab. 20, 4 relevés) is incomplete. The constancy has to be calculated on the basis of at least 5 relevés [HÁJKOVÁ \& al. 2010], but the author indicates the constancy of the species based on 4 relevés, and when describing the given association, he lists Festuca pratensis, Poa 
Pavel PÎNZARU \& al.

pratensis, Juncus articulatus, Symphytum officinale, Ranunculus acris and Lythrum salicaria as frequent species, but, in fact, they are absent in the 4 described relevés. The phytocoenoses included by LAZU (2014) in the association Cirsietum cani Tx. 1951, we include in the new association Geranio pratensi-Cisietum cani, containing the following common species: Cisium canum, Geranium pratense, Inula helenium, Filipendula ulmaria, Taraxacum camylodes (= T. officinale), Althaea officinalis, Lathyrus pratensis, Valeriana officinalis, Trifolium pratense, Angelica sylvestris. Unfortunately, the localities from where these relevés were made are not indicated, being indicated only the Central Codrii area.

The new association is described below.

\section{Ass. Geranio pratensi-Cirsietum cani}

Pînzaru, Ioniţa et Jardan, ass. nova, h. 1., Figure 2, 3, 4

Syn.: Cirsietum cani Tx. 1951: Lazu, 2014

Relevé type h. 1.: Table 1, rel. 22.

Table synthetic h. 1.: Table 1, 26 relevés

The total area of the association in the described locations is about 30 ha.

Locations: Altitude 135-150 m. Relief: Central Moldavian Plateau, in floodplains of rivers. Soils - alluvial, stratified, slightly alkaline. Climate - temperate-continental, the average annual temperature is $10.0-10.5^{\circ} \mathrm{C}$, the average annual precipitation varies between $650 \mathrm{~mm}$ and $700 \mathrm{~mm}$.

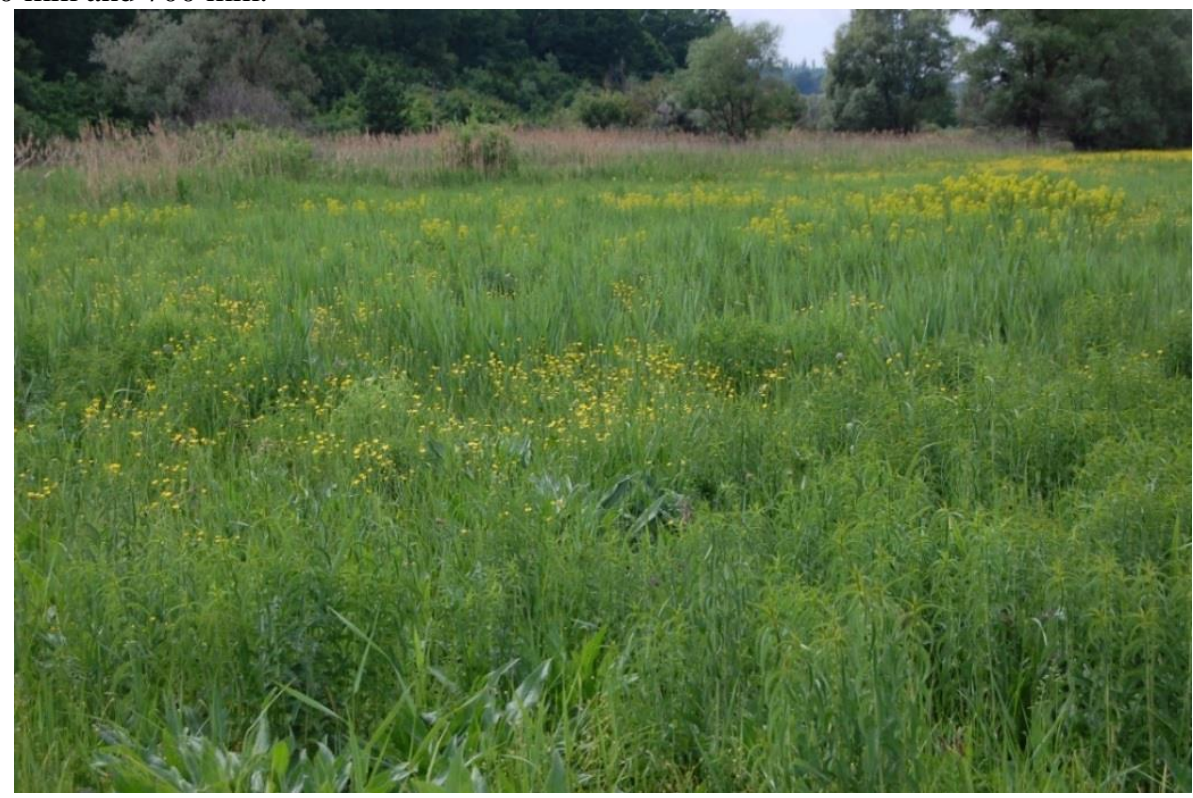

Figure 2. Ass. Geranio pratensi-Cirisetum cani - 29 May 2018, Corneşti commune 
GERANIO PRATENSI-CIRSIETUM CANI ASS. NOVA PÎNZARU, IONIȚA \& JARDAN...

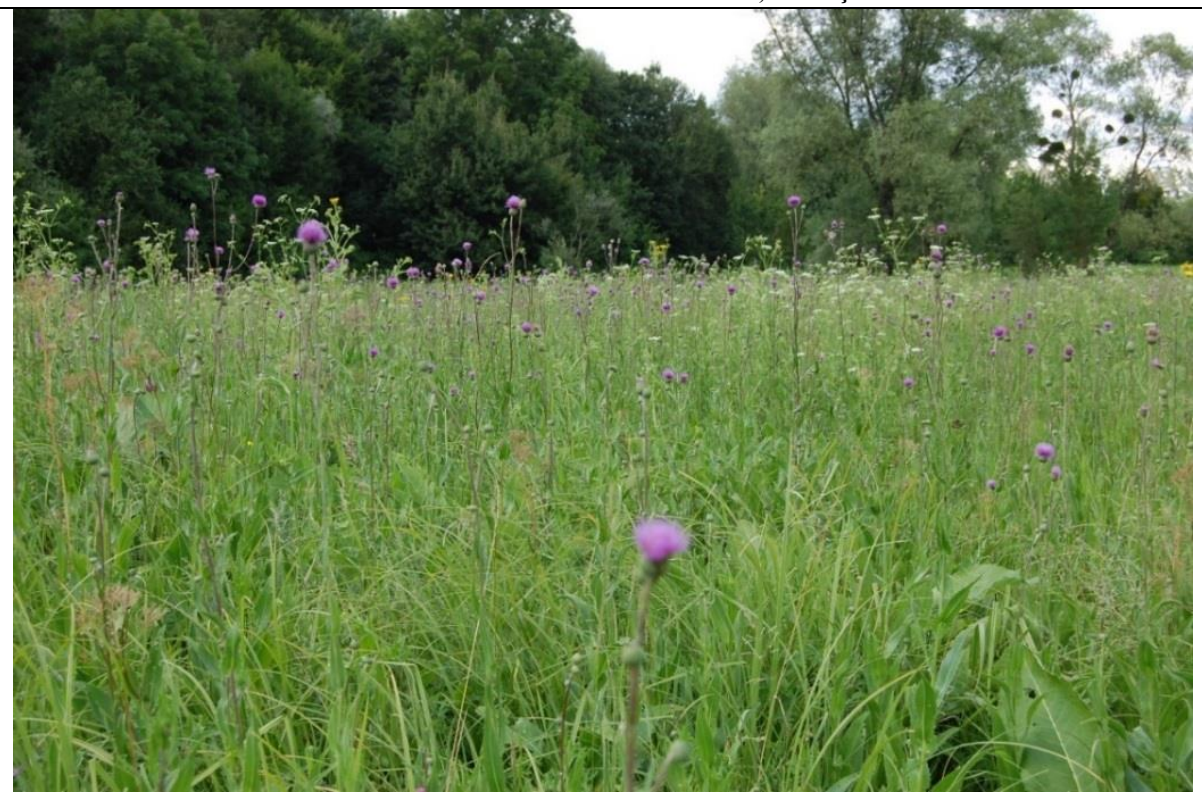

Figure 3. Geranio pratensi-Cirsietum cani ass. nova (type) - 12 July 2019, "Codru" Scientific Reserve

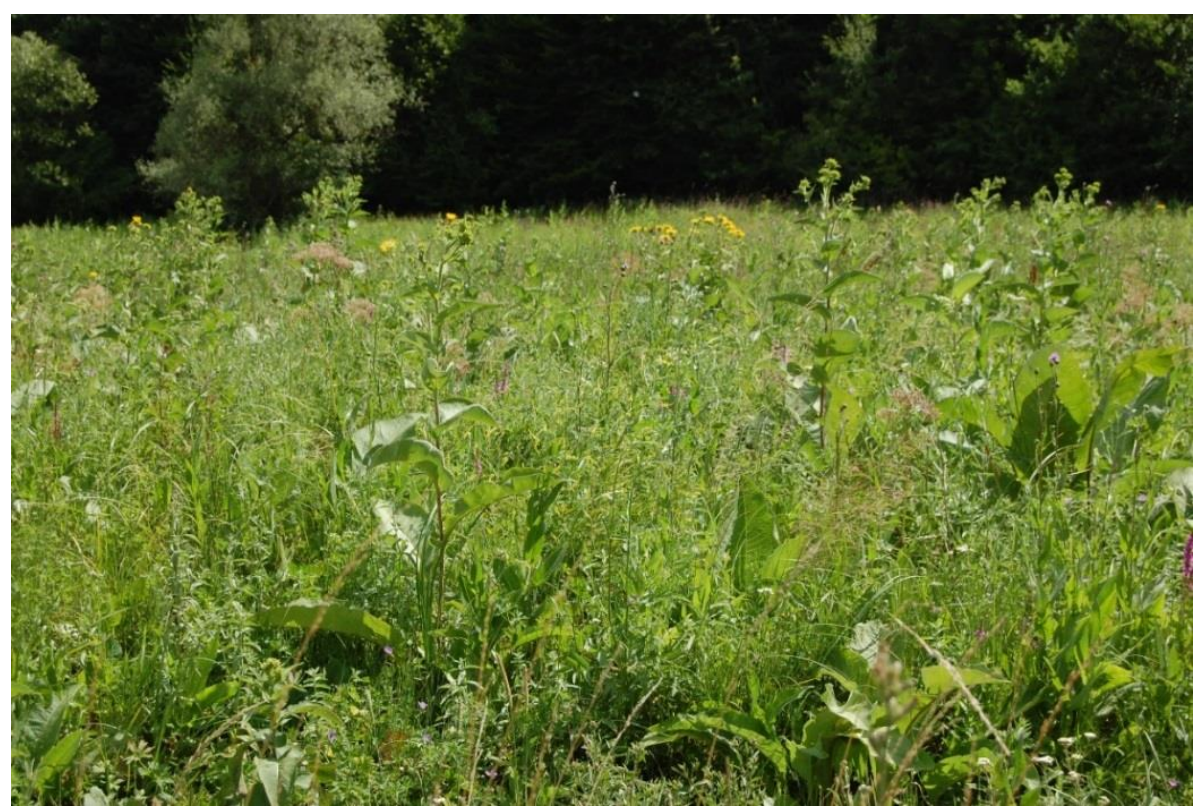

Figure 4. Ass. Geranio pratensi-Cirsietum cani-12 July 2019, "Codru" Scientific Reserve 
Pavel PÎNZARU \& al.

Characteristic species: Cirsium canum, Geranium pratense, Inula helenium.

Constant species: Valeriana officinalis, Symphytum officinale, Veronica longifolia, Thalictrum lucidum, Taraxacum camylodes, Lathyrus pratense, Achillea pannonica.

Rare species: Anacamptis palustris (= Orchis palustris) [Endangered (EN)], included in the Red Book of R. Moldova, Dactylorhiza incarnata (= D. majalis auct.mold. non (Rchb.) P. F. Hunt et Summ.) [Critically Endangered (CR)], Ophioglossum vulgatum [Critically Endangered (CR)], included in the Red Book of R. Moldova, Thelypteris confluens $(=T$. palustris) [Endangered $(\mathrm{EN})$ ], included in the Red Book of R. Moldova, Epipactis helleborine [Vulnerable (VU)], Ranunculus binatus [Vulnerable (VU)], Silene floscuculi [Endangered (EN)], Senecio sarracenicus (= S. fluviatilis ) [Critically Endangered (CR)], Galium rivale (Sibth. \& Sm.) Giseb. [Critically Endangered (CR)] [12-14].

Structure: the herbaceous layer has $100 \%$ coverage (Figure 2-4). Vertically, three layers are distinguished in phytocoenoses:

1. The upper layer, about $140-180 \mathrm{~cm}$ in height, consists of the species: Cirsium canum, Inula helenium, Thalictrum lucidum, Lysimachia vulgaris, Heracleum sibiricum, Filipendula ulmaria, Veronica longifolia, Dactylis glomerata, Valeriana officinalis, Sium sisarum, Angelica sylvestris, Senecio erucifolius, Phleum pratense, Elymus repens, Festuca arundinacea.

2. The second, middle layer, $35-110 \mathrm{~cm}$ in height, consists of Geranium pratense, Serratula tinctoria, Lathyrus pratensis, Ranunculus acris, Carex riparia, Equisetum telmateia, Lythrum salicaria, Poa pratense, Symphytum officinale, Carex hirta, Bromus arvensis, Achillea pannonica, Vicia tenuifolia, Galium aparine, Erigeron annuus.

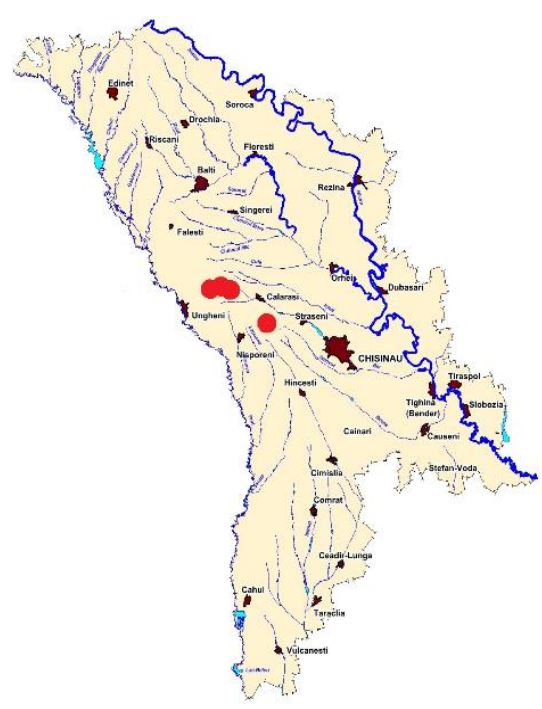

Figure 5. Locations of the ass. Geranio pratensi Cirsietum cani in the Republic of Moldova
3. The third, lower layer, which reaches up to $30 \mathrm{~cm}$ in height, is represented by Taraxacum camylodes, Ranunculus repens L., Potentilla reptans, Lysimachia nummularia, Glechoma hederacea, Veronica chamaedrys, Medicago lupulina.

Range. The plant communities of Cirsium canum with Geranium pratense occur on the Central Moldavian Plateau in the districts: Ungheni (Pojarna, Cornești), Călărași (Sipoteni), Strășeni (Lozova) (Figure 5).

Territorial protection. The phytocoenoses of this association are protected in "Codru" Scientific Reserve.

Conservation value. The given association includes phytocenoses of great value and should be protected in all the identified locations. 


\section{Conclusions}

The association Geranio pratensi-Cirsietum cani Pînzaru, Ionița et Jardan ass. nova includes phytocoenoses of tall herbaceous plants, meso-hygrophilic, which occur on slightly alkaline, stratified, alluvial soils, which are moist and rich in nutrients.

The association Geranio pratensi-Cirsietum cani Pînzaru, Ionița et Jardan ass. nova is included in the alliance Filipendulion ulmariae Segal ex Westhoff et Den Held 1969, ord. Molinietalia caeruleae Koch 1926, cl. MOLINIO-ARRHENATHERETEA Tx. 1937.

The phytocoenoses of the given association are particularly interesting from a botanical point of view. They include some very rare species in R. of Moldova, such as: Anacamptis palustris (Jacq.) R.M.Boteman, Pridegon \& M. W. Chax, Dactylorhiza incarnata (L.) Soó, Ophioglossum vulgatum L., Thelypteris confluens (Thunb.) C. V. Morton, Senecio sarracenicus L., Silene flos-cuculi (L.) Clairv., and the species Galium rivale (Sibth. \& Sm.) Giseb. and Ranunculus binatus Kit. ex Rchb. have been found only in these phytocoenoses.

We suggest to include the as. Geranio pratensi-Cirisetum cani in the List of Rare plant communities of the Republic of Moldova, with a high conservation status, and to include the sites near the communes Sipoteni (d. Călărași) and Cornești (d. Ungheni) in the network of protected areas of the Republic of Moldova. 
Pavel PÎNZARU \& al.

\begin{tabular}{|c|c|c|c|c|c|c|c|c|c|c|c|c|c|c|c|c|c|c|c|c|c|c|c|c|c|c|c|}
\hline Altitude (m) & 150 & 150 & 150 & 150 & 150 & 150 & 150 & 150 & 135 & 135 & 135 & 135 & 135 & 140 & 140 & 140 & 140 & 140 & 140 & 140 & 140 & 140 & 140 & 140 & 140 & 140 & \\
\hline $\begin{array}{l}\text { General coverage } \\
(\%)\end{array}$ & 100 & 100 & 100 & 100 & 100 & 100 & 100 & 100 & 100 & 100 & 100 & 100 & 100 & 100 & 100 & 100 & 100 & 100 & 100 & 100 & 100 & 100 & 100 & 100 & 100 & 100 & \\
\hline $\begin{array}{l}\text { Surface of relevé } \\
\left(\mathrm{m}^{2}\right)\end{array}$ & 100 & 100 & 100 & 100 & 100 & 100 & 100 & 100 & 100 & 100 & 100 & 100 & 100 & 100 & 100 & 100 & 100 & 100 & 100 & 100 & 100 & 100 & 100 & 100 & 100 & 100 & \\
\hline Number of species & 21 & 24 & 34 & 32 & 47 & 52 & 38 & 25 & 27 & 47 & 27 & 23 & 25 & 33 & 25 & 28 & 27 & 43 & 40 & 37 & 32 & 27 & 37 & 34 & 29 & 33 & \\
\hline \multicolumn{28}{|l|}{$\frac{\text { Characteristic }}{\text { species }}$} \\
\hline Cirsium canum & 3 & 3 & 1 & 2 & 3 & 2 & 4 & 4 & 2 & 3 & 1 & 3 & 2 & 3 & 3 & 2 & 2 & 2 & 1 & 1 & 2 & 3 & 3 & 2 & 4 & 4 & $\mathrm{v}$ \\
\hline Geranium pratense & 2 & 2 & 1 & 1 & 2 & 3 & 3 & 2 & 2 & 2 & 3 & 2 & 1 & 2 & + & + & 1 & 2 & 2 & 2 & 2 & 3 & 2 & 2 & 3 & 3 & $\mathrm{v}$ \\
\hline Inula helenium & - & - & + & - & 1 & 2 & + & + & 3 & + & 2 & + & - & + & + & - & 3 & 3 & 2 & 4 & 3 & 1 & 1 & + & - & + & IV \\
\hline \multicolumn{28}{|l|}{ Filipendulion } \\
\hline Valeriana officinalis & - & - & + & + & + & + & - & - & 1 & + & + & + & - & - & + & - & + & 1 & + & 1 & + & + & + & + & + & + & IV \\
\hline Veronica longifolia & 1 & 1 & + & 1 & + & + & 1 & + & 2 & 2 & 2 & 2 & - & + & - & - & - & - & - & - & - & - & - & - & - & - & III \\
\hline Thalictrum lucidum & - & - & + & + & + & + & 1 & + & - & + & + & + & - & + & - & - & - & + & - & + & - & + & - & - & - & 1 & III \\
\hline Filipendula ulmaria & - & - & - & - & - & - & - & - & - & 2 & - & - & - & - & - & - & - & + & 2 & - & - & + & 1 & + & - & - & II \\
\hline Lysimachia vulgaris & - & - & - & - & - & - & + & - & - & - & + & - & - & + & - & - & - & - & - & + & - & - & + & + & - & - & II \\
\hline Lythrum salicaria & - & - & - & - & - & + & + & - & - & - & + & + & + & - & + & - & - & - & - & - & - & - & + & + & + & + & II \\
\hline Equisetum telmateia & - & - & - & - & - & - & - & - & - & 1 & - & - & - & - & - & - & 1 & 3 & 3 & 2 & 2 & - & 2 & 1 & - & - & II \\
\hline $\begin{array}{l}\text { Eupatorium } \\
\text { cannabinum }\end{array}$ & - & - & - & - & - & + & - & - & - & - & - & - & - & - & + & - & 1 & + & + & - & - & - & + & + & - & - & II \\
\hline Mentha longifolia & - & - & - & - & - & 1 & - & - & + & - & - & - & + & - & + & + & + & 1 & + & - & + & + & 1 & - & 1 & 1 & II \\
\hline Epilobium hirsutum & - & - & - & - & + & + & - & - & - & - & - & - & - & - & - & - & - & - & - & - & - & - & - & - & - & - & I \\
\hline Calystegia sepium & - & - & 2 & - & - & - & + & - & - & 2 & 1 & - & - & - & - & - & - & - & - & - & - & - & - & - & - & - & I \\
\hline Stachys palustris & - & - & - & - & + & + & - & - & - & - & - & - & - & - & - & - & + & - & - & - & - & - & - & - & - & - & I \\
\hline Silene baccifera & - & - & - & - & - & - & - & - & - & - & - & - & - & - & - & - & - & - & - & + & - & - & + & - & - & - & I \\
\hline Euphorbia lucida & - & - & - & - & - & + & - & - & - & 2 & - & - & - & - & - & - & - & - & - & - & - & - & - & - & - & - & I \\
\hline $\begin{array}{l}\text { Epilobium } \\
\text { tetragonum }\end{array}$ & - & - & - & - & + & + & - & - & - & - & - & - & - & - & - & - & - & - & - & - & - & - & - & - & - & - & I \\
\hline Elymus caninus & - & - & - & - & - & - & - & - & - & - & - & - & - & - & - & - & - & - & - & - & - & - & 1 & 1 & - & - & I \\
\hline$\frac{\text { Deschampsion }}{\text { caespitosae }}$ & & & & & & & & & & & & & & & & & & & & & & & & & & & \\
\hline $\begin{array}{l}\text { Glechoma } \\
\text { hederacea }\end{array}$ & 2 & 2 & - & 1 & - & - & - & - & - & 2 & - & - & - & - & 2 & 2 & - & - & - & - & - & - & - & - & - & - & II \\
\hline
\end{tabular}


GERANIO PRATENSI-CIRSIETUM CANI ASS. NOVA PÎNZARU, IONIŢA \& JARDAN..

\begin{tabular}{|c|c|c|c|c|c|c|c|c|c|c|c|c|c|c|c|c|c|c|c|c|c|c|c|c|c|c|c|}
\hline Phleum pratense & - & - & 1 & - & 1 & - & - & - & 1 & 1 & 2 & - & 1 & - & - & - & - & - & - & 2 & 1 & - & - & 1 & 1 & - & II \\
\hline Festuca arundinacea & - & - & - & - & - & - & - & - & - & - & - & - & - & - & - & 1 & - & 1 & - & 2 & 1 & 1 & - & + & 1 & + & II \\
\hline $\begin{array}{l}\text { Alopecurus } \\
\text { pratensis }\end{array}$ & - & - & - & - & - & - & - & - & 1 & - & - & 1 & 1 & - & - & - & - & - & - & - & 1 & + & - & - & - & - & 1 \\
\hline $\begin{array}{l}\text { Scutellarria } \\
\text { hastifolia }\end{array}$ & - & - & - & 1 & - & - & - & - & - & - & - & + & - & - & - & - & - & - & - & - & - & - & - & - & - & - & I \\
\hline Lythrum virgatum & - & - & - & - & + & - & - & - & - & - & - & - & - & - & - & - & + & + & - & - & - & + & - & - & - & - & I \\
\hline Agrostis stolonifera & - & - & - & - & - & - & - & 2 & - & - & 2 & - & 1 & - & - & - & - & - & - & + & - & - & - & 1 & - & - & I \\
\hline Festuca pratensis & - & - & - & - & - & - & - & - & - & - & - & - & 2 & - & - & 2 & - & - & - & - & - & - & - & - & - & - & I \\
\hline \multicolumn{28}{|l|}{$\underline{\text { Molinion }}$} \\
\hline Stachys officinalis & - & - & - & + & 1 & - & - & - & - & - & - & - & - & - & - & - & - & + & - & - & + & + & + & + & + & - & II \\
\hline Serratula tinctoria & + & - & - & 2 & - & - & - & - & - & 1 & 1 & 1 & - & - & - & - & - & + & 1 & - & + & - & - & - & - & - & II \\
\hline $\begin{array}{l}\text { Anacamptis } \\
\text { palustris }\end{array}$ & - & - & - & - & - & - & - & - & - & $\mathrm{r}$ & - & - & - & - & - & - & - & - & - & - & - & - & - & - & - & - & I \\
\hline $\begin{array}{l}\text { Dactylorhiza } \\
\text { incarnata }\end{array}$ & - & - & - & - & - & - & - & - & - & $\mathrm{r}$ & - & - & - & - & - & - & - & - & - & - & - & - & $\mathrm{r}$ & - & - & $\mathrm{r}$ & I \\
\hline $\begin{array}{l}\text { Ophioglossum } \\
\text { vulgatum } \\
\underline{\text { Molinietalia }}\end{array}$ & - & - & - & - & - & - & - & - & - & - & - & - & - & - & - & - & - & - & - & 1 & - & - & - & - & - & - & I \\
\hline $\begin{array}{l}\text { Symphytum } \\
\text { officinale }\end{array}$ & + & + & 1 & + & + & 1 & 1 & 1 & + & 1 & - & - & - & 1 & + & - & - & + & + & - & + & + & + & + & + & + & IV \\
\hline Ranunculus repens & 1 & - & 2 & 1 & - & - & - & 2 & 3 & 2 & - & - & - & - & 2 & - & - & 1 & 2 & 2 & - & - & 2 & - & - & 2 & III \\
\hline Angelica sylvestris & - & - & - & - & + & + & + & - & 1 & + & + & + & - & - & + & - & + & + & + & 1 & + & + & - & - & - & + & III \\
\hline Silene flos-cuculi & - & + & 3 & 2 & - & - & - & - & - & 1 & - & - & - & + & - & - & - & - & - & - & - & - & - & - & - & - & I \\
\hline Equisetum palustre & - & - & - & - & - & - & - & - & - & - & - & - & - & - & - & - & - & - & - & - & 2 & - & - & - & 2 & 2 & I \\
\hline $\begin{array}{c}\text { Juncus articulatus } \\
\text { Potentillo- } \\
\text { Polvgonetalia }\end{array}$ & - & - & - & - & - & - & - & - & - & - & - & - & - & - & - & - & - & - & - & - & - & - & - & - & - & 2 & I \\
\hline Potentilla reptans & 1 & 2 & 2 & 1 & 2 & 2 & 2 & 2 & 2 & - & - & - & 2 & 2 & - & - & - & 2 & - & - & - & 2 & 2 & - & 2 & 2 & IV \\
\hline Elymus repens & - & - & - & - & - & 2 & 2 & 2 & - & 2 & 2 & - & 2 & - & - & 2 & 2 & - & 2 & 1 & 3 & 1 & 2 & - & - & - & III \\
\hline Althaea officinalis & - & - & - & - & + & + & - & + & - & + & - & - & - & - & - & - & - & $\mathrm{r}$ & - & - & - & - & - & - & + & + & II \\
\hline Carex hirta & - & - & 2 & - & - & - & - & - & - & - & - & - & - & - & - & - & - & - & 2 & 2 & - & 2 & 3 & 2 & 2 & 2 & II \\
\hline Rumex crispus & - & - & - & + & - & - & + & - & - & - & + & - & - & - & - & + & + & + & + & + & + & - & + & - & - & - & II \\
\hline Dipsacus laciniatus & - & - & + & - & + & + & + & - & - & - & + & + & - & - & - & - & - & + & - & - & - & - & - & - & + & - & II \\
\hline Potentilla anserina & - & - & - & - & - & 1 & - & - & - & - & - & - & + & - & - & - & - & - & - & - & - & - & 1 & - & 2 & 2 & I \\
\hline Rorippa sylvestris & - & - & - & - & - & + & - & - & - & - & - & - & - & - & - & - & - & - & - & - & - & - & + & - & - & - & I \\
\hline
\end{tabular}


Pavel PÎNZARU \& al.

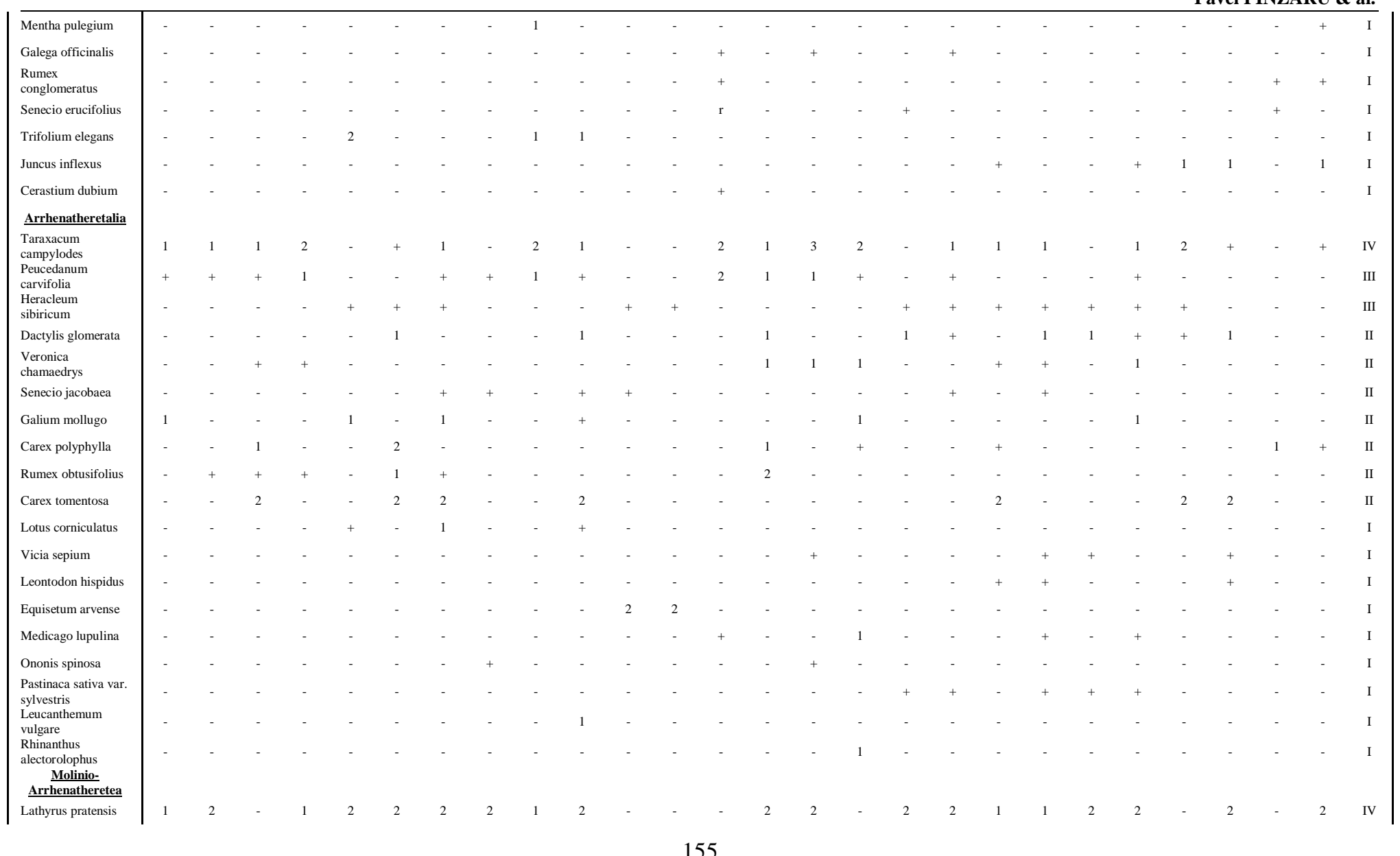


GERANIO PRATENSI-CIRSIETUM CANI ASS. NOVA PÎNZARU, IONIŢA \& JARDAN..

\begin{tabular}{|c|c|c|c|c|c|c|c|c|c|c|c|c|c|c|c|c|c|c|c|c|c|c|c|c|c|c|c|}
\hline Achillea pannonica & 1 & 1 & - & - & 1 & - & 1 & - & - & - & 1 & + & - & 1 & 1 & 1 & 1 & 1 & 1 & 1 & 1 & 1 & 1 & + & 1 & 1 & IV \\
\hline Ranunculus acris & + & 2 & - & 2 & - & 1 & 2 & 3 & 1 & - & 1 & - & 1 & 3 & 1 & 1 & 2 & - & + & - & - & - & - & - & - & - & III \\
\hline $\begin{array}{l}\text { Lysimachia } \\
\text { nummularia }\end{array}$ & - & 3 & 2 & - & 3 & - & - & - & - & 3 & - & - & - & - & - & 2 & - & 2 & 2 & 2 & 2 & 2 & 2 & 2 & - & 2 & III \\
\hline Centaurea jacea & - & - & - & - & + & + & + & - & - & + & - & - & - & - & - & - & 1 & 1 & 1 & - & 1 & 1 & - & + & - & + & III \\
\hline Poa pratensis & - & - & 2 & 2 & & 2 & - & - & - & - & - & - & - & 2 & - & - & - & - & 2 & - & - & - & 2 & - & - & - & II \\
\hline Allium oleraceum & - & - & - & - & + & - & - & - & - & - & - & - & - & - & - & - & + & - & - & - & + & + & - & + & - & + & II \\
\hline Prunella vulgaris & - & - & - & - & - & - & + & - & - & - & - & - & - & - & - & - & - & - & - & - & - & + & - & + & - & - & I \\
\hline Trifolium pratense & - & - & - & - & - & - & 2 & - & - & - & - & - & + & - & - & - & - & - & - & - & + & - & 1 & 1 & - & - & I \\
\hline $\begin{array}{l}\text { Cerastium } \\
\text { holosteoides }\end{array}$ & - & + & + & - & - & - & - & - & - & - & - & - & - & + & - & - & - & - & - & - & - & - & + & - & - & - & I \\
\hline Myosotis arvensis & - & - & - & - & - & + & - & - & - & - & - & - & - & - & - & - & - & - & + & - & - & - & - & - & - & - & I \\
\hline Trifolium repens & 1 & - & - & - & - & - & - & - & - & - & - & - & - & - & 2 & 3 & - & - & - & - & - & - & - & - & 2 & 1 & I \\
\hline Ranunculus binatus & 1 & 2 & - & 2 & - & - & - & - & - & 1 & - & - & - & 1 & - & - & - & - & - & - & - & - & - & - & - & - & I \\
\hline $\begin{array}{l}\text { Ranunculus stevenii } \\
\text { Phragmitetea }\end{array}$ & - & - & - & - & - & - & - & - & - & - & - & - & - & - & - & + & - & - & - & - & - & - & + & - & - & - & I \\
\hline Iris pseudacorus & + & + & + & + & + & 2 & 1 & 1 & 1 & 1 & 1 & + & - & 1 & - & - & - & - & - & - & - & - & - & - & - & - & III \\
\hline Carex riparia & - & - & 2 & 1 & 2 & 2 & - & 2 & 2 & + & 2 & 2 & - & - & - & - & 3 & 2 & 2 & 2 & - & - & - & - & 1 & 2 & III \\
\hline Phalaris arundinacea & - & - & - & 2 & 1 & 2 & 1 & 1 & 1 & 1 & - & 2 & - & - & - & - & - & 1 & 2 & 1 & - & 1 & - & 1 & 1 & + & III \\
\hline Sium sisarum & - & - & - & - & + & - & - & - & - & - & - & - & - & - & - & + & + & + & + & + & + & + & + & - & + & 1 & III \\
\hline Phragmites australis & - & - & - & 1 & - & - & - & - & - & + & - & - & - & + & - & - & - & 1 & - & - & - & - & 2 & - & - & - & I \\
\hline $\begin{array}{l}\text { Thelypteris } \\
\text { confluens }\end{array}$ & - & - & - & - & - & - & - & - & - & - & - & - & - & - & - & - & - & - & - & - & - & - & 1 & - & - & - & I \\
\hline Lycopus exaltatus & - & - & - & - & - & + & - & - & - & - & - & - & - & - & - & - & - & - & - & - & - & - & - & - & - & - & I \\
\hline Senecio saracenicus & - & - & - & - & - & $\mathrm{r}$ & - & - & $\mathrm{r}$ & - & - & - & - & - & - & - & - & - & - & - & - & - & - & - & - & - & I \\
\hline $\begin{array}{l}\text { Glyceria } \\
\text { arundinacea }\end{array}$ & - & - & - & - & - & - & - & - & - & - & - & - & - & - & - & - & - & - & - & - & - & - & - & 2 & - & - & I \\
\hline $\begin{array}{l}\text { Carduus crispus } \\
\text { Variae syntaxa }\end{array}$ & - & - & - & - & - & - & - & - & 1 & - & - & - & - & - & - & - & - & - & - & - & - & - & 1 & - & - & - & I \\
\hline Erigeron annuus & - & - & + & - & - & + & + & - & + & - & + & + & - & - & + & - & - & + & + & + & - & - & - & + & + & - & III \\
\hline Galium verum & - & 1 & - & + & 1 & + & - & + & 1 & + & + & - & + & - & - & + & - & + & - & - & - & - & - & 1 & - & - & III \\
\hline Galium aparine & 1 & - & 2 & 1 & - & - & - & - & - & - & - & - & - & 1 & - & 1 & 2 & 2 & 2 & 2 & 2 & - & 1 & 2 & - & - & III \\
\hline Daucus carota & - & - & + & & + & - & + & + & + & + & - & - & + & - & - & - & - & + & - & - & + & + & - & - & + & - & II \\
\hline
\end{tabular}


Pavel PÎNZARU \& al.

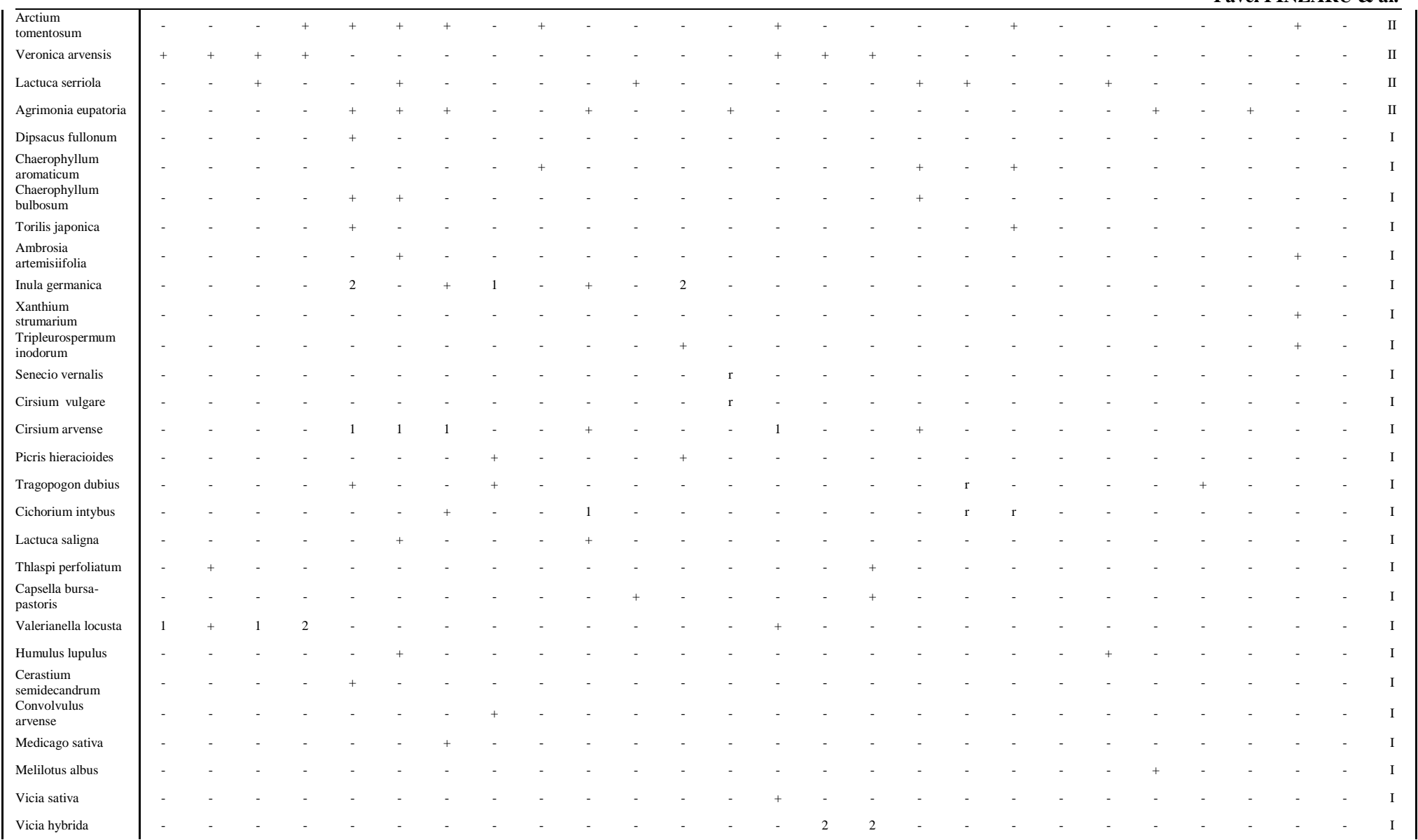


GERANIO PRATENSI-CIRSIETUM CANI ASS. NOVA PÎNZARU, IONIŢA \& JARDAN..

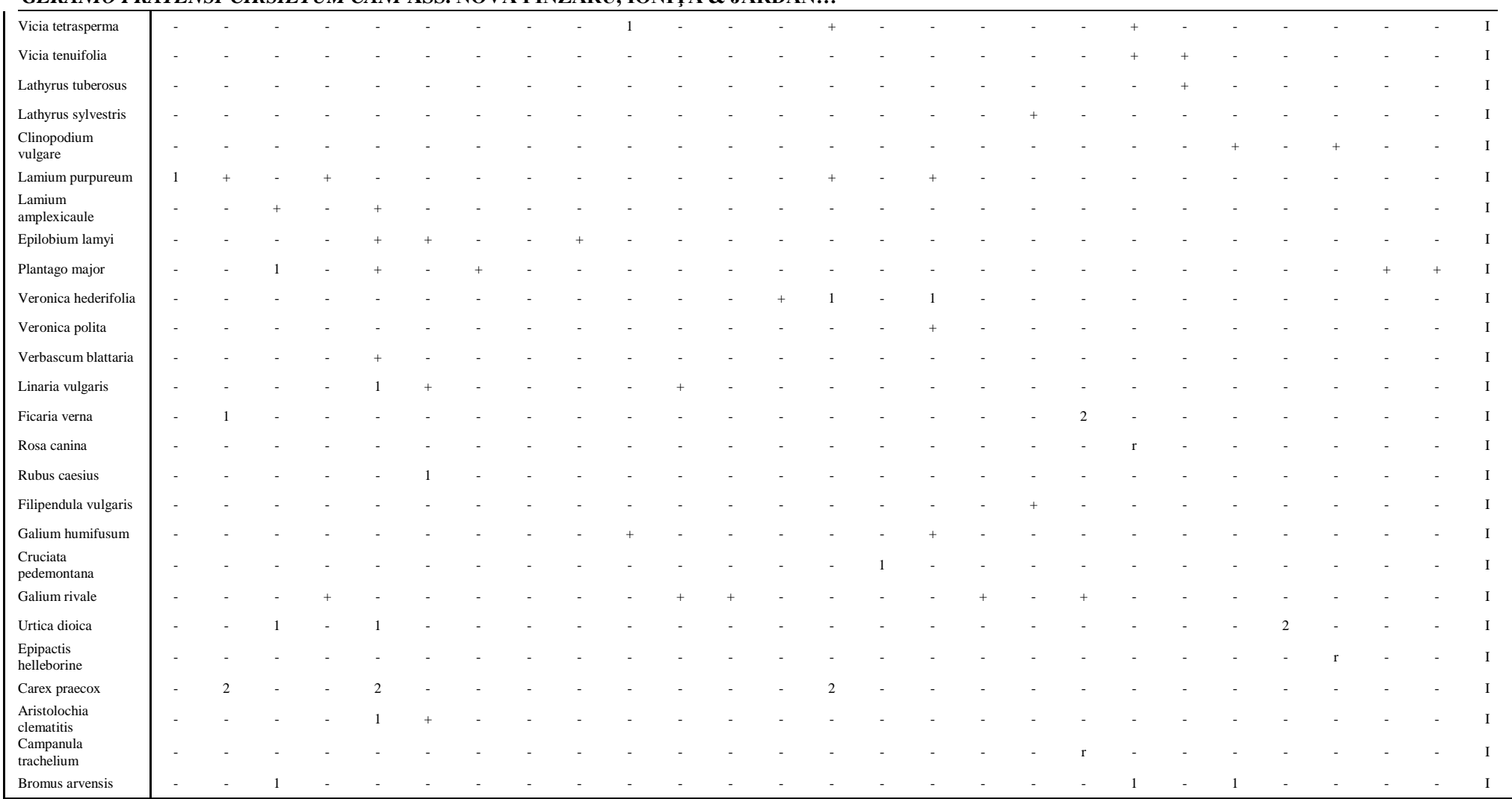

Place and date of the relevés: 1-8, Cornești commune, Ungheni district, 07.VII.2018, 11.VIII.2018; 04.V.2019; 9-13, Sipoteni commune, Călăras district, 04.V.2019; 14-26 (*22 -typus), Lozova commune, Strășeni district, 15.V.2019, 12.VII.2019. 


\section{References}

AESCHIMANN D., LAUBER K., MOSER D. M. \& THEURILLAT J. P. 2004. Flora alpina. Vol. 2. GentianaceaeOrchidaceae. Bologna: Zanichelli, $1159 \mathrm{pp}$.

BĂDĂRĂU A. S. \& ALEC-FARCAS A. 2010. The halophyte vegetation with Peucedanum latifolium (M. Bieb.) DC. from the Transylvania. In: Studii și cercetări, Biology, Bistrița. 15: 5-16.

BRAUN-BLANQUET J. 1964. Pflanzensoziologie. Grundzüge der Vegetationskunde. Ed. 3. Wien: Springer Verlag, $855 \mathrm{pp}$.

CHIFU T. \& ZAMFIRESCU O. 2014. Molinio-Arrenatheretea R. Tx. 1937: 398-629. In: CHIFU T. (ed.), IRIMIA I. \& ZAMFIRESCU O. Diversitatea fitosociologică a vegetației României. II. Vegetaţia erbacee antropizată. A. Vegetația pajiştilor. Iași: Institutul European. ISBN 978-606-24-0090-3, 659 pp.

COLDEA G. 2012. Molinio-Arrenatheretea R.Tx. 1937. Pp. 191-234. In: COLDEA G. (ed.), OPREA A., SÂRBU I., SÎRBU C. \& ȘTEFAN N. Les associations végétales de Roumanie. Tome 2. Les associations anthropogènes. Cluj-Napoca: Presa Universitară Clujeană, ISBN 978-973-595-372-0, 482 pp.

CRISTEA V., GAFTA D. \& PEDROTTI F. 2004. Fitosociologie. Cluj-Napoca: Presa Universitară Clujeană, ISBN 073-610-192-4, 394 pp.

HÁJKOVÁ P., HÁJEK M., BLAŽKOVÁ D. \& al. 2010. Třídu TD Molinio-Arrhenatheretea Tüxen 1937: $165-280$. In: CHYTRÝ M. (ed.) Vegetace České republiky. 1. Travinná a keřičková vegetace. = Vegetation of the Czech Republic. 1. Grassland and Heathland Vegetation. Praha: Academia, ISBN 978-80-200-1896-0, $528 \mathrm{~s}$.

LAZU Ș. 2014. Pajiștile de luncă din Republica Moldova. Chișinău: Tipografia AȘM, ISBN 978-9975-62-377-3. $452 \mathrm{pp}$.

MUCINA L., BULTMANN H., DIERßEN K. \& al. 2016. Vegetation of Europe: hierarchical floristic classification system of vascular plant, bryophyte, lichen, and algal communities. In: Applied Vegetation Science 19. Suppl. 1: 3-264.

NEDEALCOV M. RĂILEAN V., CHIRICĂ L. \& al. 2013. Atlas. Resursele climatice ale Republicii Moldova = Atlas. Climatic resources of the Republic of Moldova. Chișinău: Î. E. P. Știința, 76 pp.

PÎNZARU P. 1996. Asociația Petasitetum hybridi (Dostal 1933) Soó 1940 în Rezervația de Stat „Codrii”. In: Rezumatele lucrărilor Simpozionului jubiliar Rezervația naturală Codrii -25 de ani. Realizări, probleme, perspective. 19-20 sept. 1996. Comuna Lozova, 178-180.

PÎNZARU P. \& CANTEMIR V. 2018. Floristic notes in Bessarabia no. 165-200. In: Journal of Botany. Chisinau, vol. X, 2(17): 32-41.

PÎNZARU P. \& JARDAN N. 2019. Ophioglossum vulgatum L. (Ophioglossaceae) - specie nouă în flora Rezervației ştiințifice „Codrii” din Republica Moldova. In: Mat. Conf. șt. naționale cu participare internațională $27-$ 28 septembrie 2019. „Invățământ superior, tradiții, valori, perspective”. Vol. 1. Șt. Exacte şi ale Naturii şi Didactica Șt. Exacte şi ale Naturii. Chișinău: Tipografia UST: 45-48.

PÎNZARU P. \& SÎRBU T. 2016. Flora vasculară din Republica Moldova (Lista speciilor și ecologia). Chișinău: Tipografia UST, ISBN 978-9975-76-185-7, 261 pp.

SÂRBU I., ȘTEFAN N. \& OPREA A. 2013. Plante vasculare din România. Determinator ilustrat de teren. București: Edit. Victor B Victor, 1317 pp.

*** 2015. Cartea Roşie a Republicii Moldova = The Red Book of the Republic of Moldova, ed. 3. Col. Red. DUCA G. \& al. Chișinău: Știința, Part. I: 11-231.

${ }^{* * * *}$ Legea privind fondul ariilor naturale protejate de stat. Nr. 1538 din 25.02.1998. Monitorul Oficial din 16.07.1998, Nr. 066 art. Nr. 442.

\footnotetext{
How to cite this article:

PÎNZARU P., IONIȚA O. \& JARDAN N. 2019. Geranio pratensi-Cirsietum cani ass. nova Pînzaru, Ionița \& Jardan (Filipendulion Segal ex Westhaff et Den Held 1969) in the Republic of Moldova. J. Plant Develop. 26: 147-159. https://doi.org/10.33628/jpd.2019.26.1.147
} 